\title{
An Oscillatory Neural Network for Image Segmentation
}

\author{
Dênis Fernandes ${ }^{1}$ and Philippe Olivier Alexandre Navaux ${ }^{2}$ \\ ${ }^{1}$ PUCRS - Faculdade de Engenharia - Pontifícia Universidade Católica do Rio Grande do Sul, \\ Av. Ipiranga, 6681, 90619-900 Porto Alegre, RS, Brazil \\ denis@ee.pucrs.br \\ ${ }^{2}$ UFRGS - Instituto de Informática - Universidade Federal do Rio Grande do Sul, Av. Bento \\ Gonçalves, 9500, 91501-970 Porto Alegre, RS, Brazil \\ navaux@inf.ufrgs.br
}

\begin{abstract}
Oscillatory neural networks are a recent approach for applications in image segmentation. Two positive aspects of such networks are its massively parallel topology and the capacity to separate the segments in time. On the other hand, limitations that restrict the practical application are found in the proposed oscillatory networks, such as the use of differential equations, implying high complexity for implementation in digital hardware, and limited capacity of segmentation. In the present paper, an oscillatory neural network suitable for image segmentation in digital vision chips is presented. This network offers several advantages, including unlimited capacity of segmentation. Preliminary results confirm the successful operation of the proposal in image segmentation and its good potential for real time video segmentation.
\end{abstract}

\section{Introduction}

The increasing demand for artificial vision systems, which implement complex algorithms with high speed, justifies the development of vision chips [10] or silicon retinas [3]. In these chips, the photo detectors corresponding to the pixels of the image are jointly integrated with a massively parallel network of processing elements (PEs) for execution of specific operations over the input image. Analog implementation of vision chips requires simpler circuits than the digital one, presenting, on the other hand, lower flexibility to reprogram the executed function [10]. So, when flexibility is required, the digital implementation is more attractive.

Recently, alternative topologies of artificial neural networks, the oscillatory neural networks, which are inspired on the mechanism of segmentation executed by the human brain, have been applied in image segmentation with satisfactory results [4][6][11][14]. The study of these networks is a fertile field of work, as well as the development of dedicated architectures for efficient implementation [2][6].

The LEGION network (Locally Excitatory Globally Inhibitory Oscillator Network) [15] is the most consistent proposal of oscillatory neural network for image segmentation found in the bibliography. Its applications include segmentation of remote sensing images [12], medical images [8][13], and electronic microscope images [7]. An interesting aspect of this network is the capability to separate the segments of the image in time, facilitating later identification and quantification. Its massively parallel nature is adequate to the implementation of vision chips for image segmentation. The negative aspects include high computational complexity for implementation in digital 
hardware as a consequence of the use of differential equations, limited segmentation capacity, and also the high amount of parameters and their little intuitive setting.

This paper introduces a new model of oscillatory neural network inspired on LEGION network, suitable to applications of image segmentation and implementation in vision chips with digital technology. The network presents massively parallel topology and it is able to separate the segments in time. The low complexity and the non-limitation regarding the number of segments to be discriminated are advantages of the network. The use of few parameters with intuitive setting is also a positive aspect, as well as the reduced number of iterations and the easy predictability of the time to reach the results. The digital structure also presents flexibility for easy implementation of more sophisticated segmentation procedures, using different attributes of the image. Results found in practical implementations are presented, proving that the operation of the proposed network was performed according to the expectations, and showing its potential efficiency to real time video segmentation.

\section{The Proposed Oscillatory Neural Network}

In the late 80's, oscillations of approximately $40 \mathrm{~Hz}$ were discovered in the visual cortex of the human brain. Such oscillations have strong correlation with the visual stimulus and synchronism of phase occurs between physically near neurons that receive similar stimulus, which can characterize a homogeneous region of the perceived image. Physically near neurons that receive different stimulus or physically distant neurons do not present such synchronism of phase [4][16].

A new oscillatory neural network was conceived [9] using the property of local synchronism between neurons and adding a mechanism of global inhibition implemented with local connections to get anti-synchronism among different groups of neurons. The proposed network is suitable to application in image segmentation and implementation in digital hardware with massively parallel topology.

\subsection{Structures of Connections}

The proposed network is implemented in a two-dimensional topology with the same size of the image to be segmented. Two structures of connections among neurons, called excitatory connections and inhibitory connections, are used.

Figure 1 $(a)$ presents an example of the excitatory connection structure. A neuron has its excitatory output $v_{e}$ simultaneously connected to the 8 nearest neurons. So, the excitatory output of a neuron will be activated when at least one of its nearest neighbors, with similar input, is active.

Figure 1(b) presents an example of the inhibitory connections structure adopted. Each neuron has its inhibitory output $v_{i o}$ connected to only one neighboring neuron. This structure establishes a priority order and neurons with higher priority inhibit the remainders. The excitatory connections will cause the only neuron qualified by the inhibitory connections to excite the other neurons belonging to same segment. The inhibitory output of the neuron with the lowest priority will be active only when at least one neuron is active. In a practical implementation, this signal can be used to detect images without any segment present at the output of the network. 


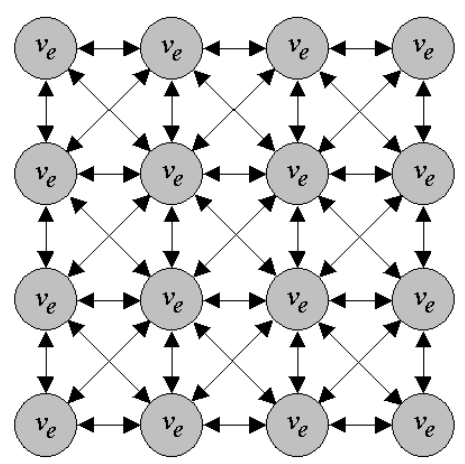

(a)

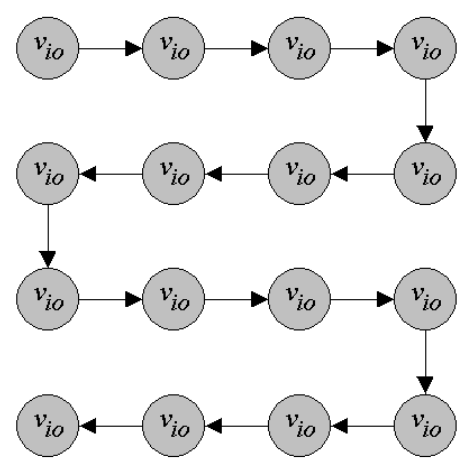

(b)

Fig. 1. Examples of structures of excitatory connections $(a)$ and inhibitory connections $(b)$

\subsection{The Network Neuron}

The basic idea for the conception of the proposed neuron consists of associating a binary counter to each of them in a way that neighboring neurons with similar inputs are synchronized in the same state, different from the states of the other groups (Figure 2). The neurons stay inactive, until its counters reach a predefined state.

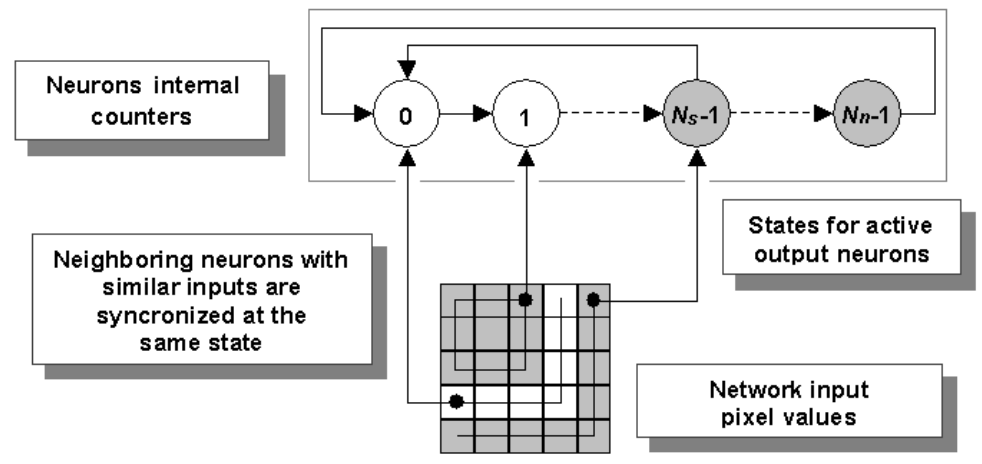

Fig. 2. Basic idea behind the conception of the proposed neuron

Figure 3 presents the internal logic structure of the proposed neuron. The definitions of the constants and variables are listed below:

- $N_{n}$ : total number of neurons in the network;

- $N_{s}$ : maximum number of segments allowed to be separated by the network;

- $L_{w}$ : threshold for weight determination;

- $v_{c}(i, j, t)$ : state of the internal binary counter belonging to the neuron placed at line $i$ and column $j$ in time (iteration) $t$;

- $v_{o}(i, j, t)$ : output signal of the neuron internal comparator;

- $v_{e}(i, j, t)$ : excitatory output of the neuron; 
- $v_{i o}(i, j, t)$ : inhibitory output of the neuron;

- $v_{i i}(i, j, t)$ : inhibitory input of the neuron;

- $v_{l}(i, j, t)$ : leader indication signal;

- $I_{a}(i, j, t)$ : input signal of the neuron representing a set of features of the related pixel;

- $w(i, j, k, l, t)$ : weight related to the comparison between the input of the neuron placed at line $i$ and column $j$ and the input of the neuron placed at line $k$ and column $l$.

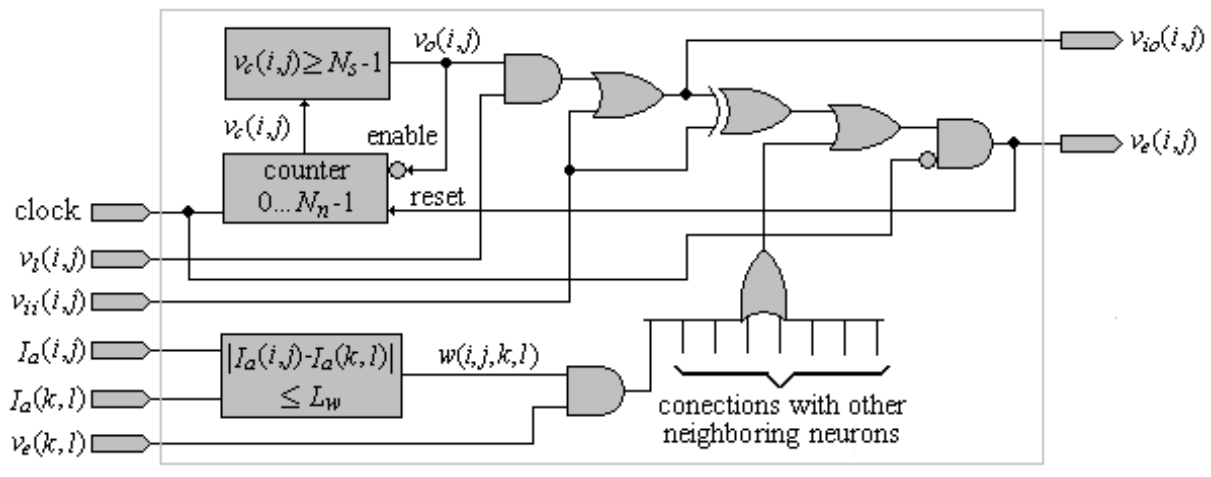

Fig. 3. Neuron internal structure of the proposed network

A binary counter with $N_{n}$ states defines the neuron state as a function of the time. The counter receives a synchronous reset if the neuron is active $\left(v_{e}(i, j, t)=1\right)$, otherwise its state is increased until $N_{s}-1$, remaining there until it is reset. The use of $N_{n}$ states makes the discrimination of a maximum of $N_{n}$ segments possible, even though, in this case, there is no similarity between any inputs of neighboring neurons. The proposed neuron structure qualifies the network to separate the $N_{s}$ first segments according to the sequence established by the structure of inhibitory connections.

The leader signal can be generated internally or externally and only neurons with $v_{l}(i, j, t)=1$ will be initially qualified to pass to the active phase (leaders). Neurons with $v_{l}(i, j, t)=0$ can be activated through an active neighbor with similar input. In the external generation, some criteria such as the position in the image (for example) can be established. It could be established that neurons in the central region of the image would be the only ones qualified to pass to the active phase, causing the appearance of only that segment at the output of the network. In the internal generation of the signal, one can use, for example, the criteria that a leader must have all the excitatory weights active, which correspond to a pixel in the center of a homogeneous region.

The weights of the network are determined through the comparison of each neuron input attribute intensities with the respective inputs of its neighboring neurons (1). In the cases where the differences between such inputs are, in module, below a threshold $L_{w}$, the respective weights will be unitary. Different attributes of the image can be used to carry through the segmentation. On the other hand, $I_{a}(i, j, t)$ can represent vectors of attributes related to the pixels. In this case, the weights can be determined using a measure of vectorial distance, implying more complex structures. In color images, the Euclidean distance could be used to determine the weights, which would result in segmentation by color similarity. 


$$
w(i, j, k, l, t)=\left\{\begin{array}{lll}
0 & \text { if } & \left|I_{a}(i, j, t)-I_{a}(k, l, t)\right|>L_{w} \\
1 & \text { if } & \left|I_{a}(i, j, t)-I_{a}(k, l, t)\right| \leq L_{w}
\end{array}\right.
$$

\section{Implementation and Results}

To verify the functioning of the proposed network, two types of implementation were carried out. The first one is related to the simulation of the behavior of the network through an algorithm implemented in a PC computer. The second one is the use of the Max+plusII program from Altera [1] for simulation of the network and verification of the viability of its implementation in digital devices [9].

\subsection{Segmentation of an Artificial Image}

Figure 4 presents an example carried out through the algorithm that simulates the proposed network. The input image, with $100 \times 310$ pixels, is placed at the top left position. The additional 7 images are the non-null segments got from the output of a network with $100 \times 310$ neurons. In these 7 images, each pixel is a neuron output and the white represents the active neurons. The weights were calculated on the basis of the neighboring pixel intensity differences with $L_{w}=0$. The neurons with all the excitatory weights unitary were considered leaders. The first segment is the background, having the interior parts of the characters "P" and " $\mathrm{A}$ " not presented, as they are not physically connected. All the characters were correctly isolated in time, facilitating the application of a character recognition procedure.

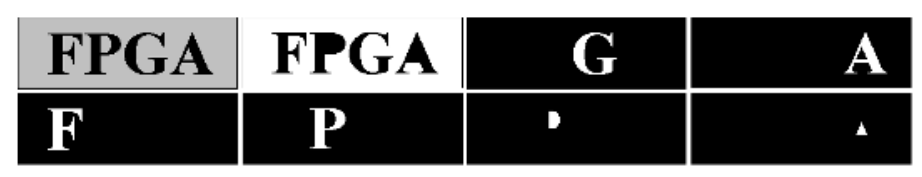

Fig. 4. Segmentation using the algorithm that simulates the proposed network operation

\subsection{A Practical Application}

Figure 5 presents a gray level image with $1482 \times 2060$ pixels and 8 bits, which was obtained through transmission electron microscopy (TEM). This image represents a silicon sample where ions of helium were implanted with the objective to reduce defects in the crystalline structure. The helium accumulates itself in bubbles concentrated in some areas of the silicon. For evaluation of the process, it is necessary to determine the gas volume in the sample. Such volume can be found through the counting of bubbles and estimation of their areas. This procedure must be carried through for several images, being a complex task for human manual execution [7]. 


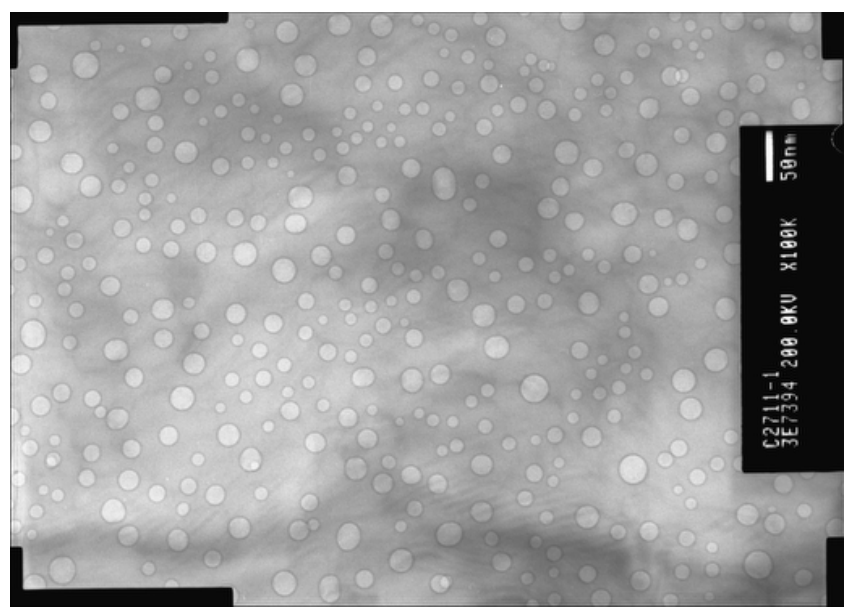

Fig. 5. Image of TEM representing a silicon sample with helium bubbles

The proposed network was used to segment the helium bubbles and simultaneously separate them in time, facilitating the implementation of an automatic process of counting and area measuring. Figure 6(a) presents a region extracted from Figure 5, which is used to verify the qualitative results of the proposed network.

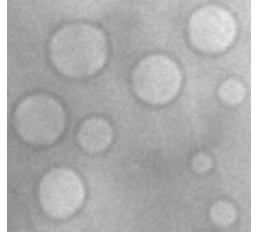

(a)

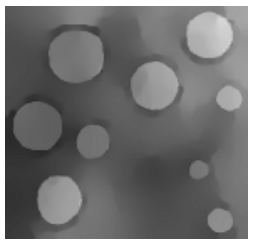

(b)

Fig. 6. Region extracted from Figure $5(a)$ and filtered with the FPS algorithm $(b)$

As other segmentation procedures, the proposed network is sensitive to noise in the input image. Such problem makes the use of a smoothing filter necessary. Figure $6(b)$ presents the result of filtering the Figure 6(a) image using the FPS (Feature Preserving Smoothing) algorithm [5]. The noise reduction and the preservation of the contours of the helium bubbles can be easily observed.

Figure 7 presents the non-null segments got from the output of a network with 1482x2060 neurons. Besides the 9 bubbles, a segment representing the silicon background was also supplied (bottom right position), which can be easily detected and discarded in the automatic procedure of measuring. The network weights were calculated on the basis of the neighboring pixel intensity differences with $L_{w}=3$.

The bubble areas can be obtained computing the ratio between the number of active neurons and the total number of neurons. Knowing the relation in pixel/nm (Figure 5), the helium volume can be estimated. Visual area determination can be inexact 


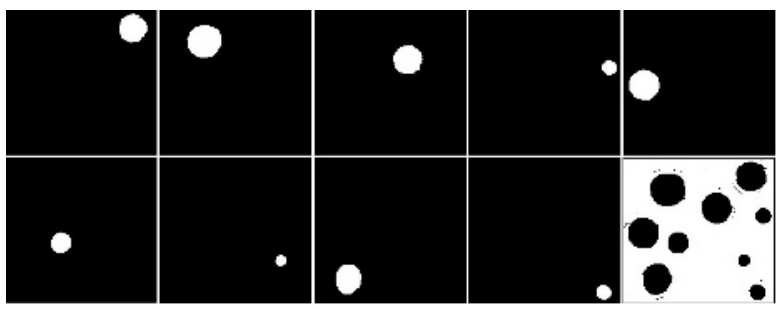

Fig. 7. Segments of Figure 6(b) image obtained with the proposed neural network

if the bubbles are not perfectly circular. Such limitation is not observed in the proposed method. Incomplete, degraded and superimposed bubbles can lead to wrong measures. For these situations, the manual process can be used.

\section{Conclusion}

The use of oscillator networks to simulate the capacity of image segmentation of the human brain is a recent proposal with good results. In this context, the LEGION network is the most consolidated model. Its massively parallel topology and the capacity to separate the segments in time are highly attractive. On the other hand, the structure based on differential equations has high complexity for digital machines. Another disadvantage is the limitation to segment a high number of objects simultaneously. The high number of parameters and their little intuitive setting are also drawbacks.

The oscillatory neural network presented by this paper has massively parallel topology and capacity to separate the homogeneous regions of the input image in time. The network does not present limitation related to the number of segments, has lower complexity, is suitable for implementation in digital vision chips, and uses few parameters, with easy setting. Other positive aspects are the easiness of synchronism and the necessity of a low and easily predictable number of iterations to get the result. The addition of a random signal to the inputs, like in the LEGION, is not necessary, which is also a factor of complexity reduction. Since the weights are obtained directly from the attributes of the input image, there is no need of training, in contrast with other neural networks. Finally, the weight determination on the basis of diverse attributes of the input image, can lead to more sophisticated segmentation procedures.

The good results obtained with the proposed network through an algorithm that simulates its behavior prove the consistency and its several positive characteristics. The simulation of a small size network and its implementation in an FPGA chip confirms the correct functioning of the proposal. Also, preliminary statistical analysis have showed that the proposed network can segment images with additive Gaussian noise and $20 \mathrm{~dB}$ of signal-to-noise ratio with less than $0.02 \%$ of misclassified pixels.

As there are no references to implementations of LEGION network using massively parallel digital hardware, the evaluation of the complexity gain of the proposed network is not possible. On the other hand, computational simulations show that the proposed network is much faster than the LEGION network version based on differential equations. Specific studies for complexity reduction are under development in order to implement practical networks using commercial FPGA devices. 
Based on the results, it is concluded that the proposed oscillatory neural network is attractive for applications of image segmentation with implementation in massively parallel digital hardware. Its several advantages imply viability for implementation of vision chips using digital technology, allowing high speed in image segmentation.

\section{References}

1. ALTERA, MAX+PLUS II Software Overview, http://www.altera.com/products/ sofware/maxplus2/mp2-index. html (20/06/2002)

2. ANDO, H., MIYAKE, M., MORIE, T., NAGATA, M., IWATA, A. A Nonlinear Oscillator Network Circuit for Image Segmentation with Double-threshold Phase Detection, ICANN 99, IEE, Edinburgh, UK, 1999, p. 655-660

3. BARBARO, M., BURGI, P., MORTARA, A., NUSSBAUM, P., HEITGER, F. A 100x100 Pixel Silicon Retina for Gradient Extraction With Steering Filter Capabilities and Temporal Output Coding, IEEE Journal of Solid-State Circuits, 2002, v.37, n.2, p.160-172

4. BUHMANN, J., von der MALSBURG, C. Sensory Segmentation by Neural Oscillators, IJCNN 91, IEEE, Seattle, USA, 1991, p. II603-II607

5. CHEN, Ke; WANG, DeLiang. Image Segmentation Based on a Dynamically Coupled Neural Oscillator Network, IJCNN 99, IEEE, Washington D. C., USA, 1999

6. COSP, J., MADRENAS, J. A Neural Network for Scene Segmentation Based on Compact Astable Oscillators, ICANN 99, IEE, Edinburgh, UK, 1999, p. 690-695

7. FERnANDES, D., NAVAUX, P. O. A., FICHTNER, P. F. P. Segmentation of TEM Images Using Oscillatory Neural Networks, SIBGRAPI 2001, IEEE, Florianópolis, Brazil, 2001, p.289-296

8. FERNANDES, D., SIQUEIRA, M. L., NAVAUX, P. O. A. Segmentation of Fetal Echocardiographic Images Using Self-Organizing Maps and Oscillatory Neural Networks, SIARP 2001, IARP, Florianópolis, Brazil, 2001, p.55-60

9. FERNANDES, D., STEDILE, J. P., NAVAUX, P. O. A. Architecture of Oscillatory Neural Network for Image Segmentation. SBAC-PAD 2002, IEEE, Vitória, Brazil, 2002

10. KOMURO, T., ISHII, I., ISHIKAWA, M. Digital Vision Chips and High-Speed Vision Systems, Symposium on VLSI Circuits Digest of Technical Papers, IEEE, Kyoto, Japan, 2001, p.1-4

11. KUROKAWA, H., MORI, S. A Local Connected Neural Oscillator Network for Sequential Character Segmentation, ICNN 97, IEEE, Texas, USA, 1997, p.838-843

12. LIU, X., CHEN, K., WANG, D. L. Extraction of hydrographic regions from remote sensing images using an oscillator network with weight adaptation, Transactions on GeoScience and Remote Sensing, IEEE, 2001, 39(1), 207-211

13. SHAREEF, N., WANG, D. L., YAGEL, R. Segmentation of Medical Images Using LEGION, Transactions on Medical Imaging, IEEE, 1999, v.18, n.1

14. TERMAN, D., WANG, D. L. Global competition and local cooperation in a network of neural oscillators, Physica D, 1995, v.81(1-2), pp.148-176

15. WANG, D., TERMAN, D. Image Segmentation Based on Oscillatory Correlation, Neural Computation, MIT, 1997, v.9, p.805-836

16. WANG, D. Relaxation Oscillators and Networks. Wiley Encyclopedia of Electrical and Electronics Engineering, Wiley \& Sons, 1999, v.18, p.396-405 